Supporting Information:

\title{
Nanomechanics of Protein Unfolding Outside a Generic Nanopore
}

\author{
Binquan Luan ${ }^{1}$, Tien Huynh ${ }^{1}$, Jingyuan $\mathrm{Li}^{2}$ and Ruhong Zhou ${ }^{1}$ \\ ${ }^{1}$ Computational Biological Center, IBM Thomas J. Watson Research, Yorktown Heights, \\ NY 10598, USA. \\ ${ }^{2}$ Key Laboratory for Biomedical Effects of Nanomaterials and Nanosafety, Institute of High \\ Energy Physics, Chinese Academy of Sciences, Beijing 100049, China.
}

1. AFM-Unzipping of two $\beta$-sheets in the partially unfolded unbiquitin

a

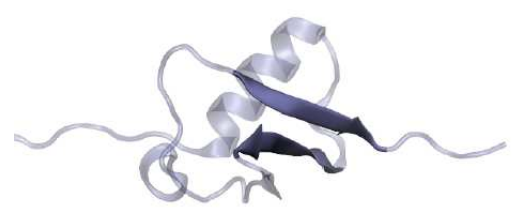

b

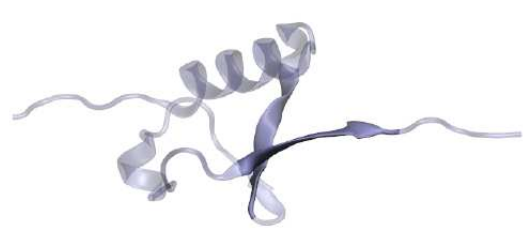

C

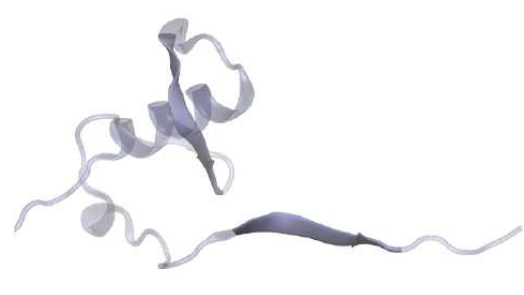

Fig. S1 The AFM-like unfolding process in unzipping two neighboring $\beta$-sheets in the partially unfolded Ubiquitin protein. (a) Two $\beta$-sheets were initially antiparallel; (b) During the unzipping process, two $\beta$-sheets were torn away from each other (tearing fracture); (c) Two $\beta$-sheets were perpendicular when being fully separated.

Figure S1 illustrates the unfolding process of two $\beta$-sheets, corresponding the second force peak (orange line; at $75 \AA$ ) in Fig. 3a. Due to the partially unfolded structure, forces exerted on both $\mathrm{N}$ - and $\mathrm{C}$ - terminals resulted in a torque on these two $\beta$-sheets. Thus, different from the shearing separation of two $\beta$-sheets signified by the first force-peak, two $\beta$-sheets of the Ubiquitin core were torn apart. Because of the tearing fracture, the required force is much smaller (see main text for details). 


\section{AFM-unfolding of the Ubiquitin core}

a

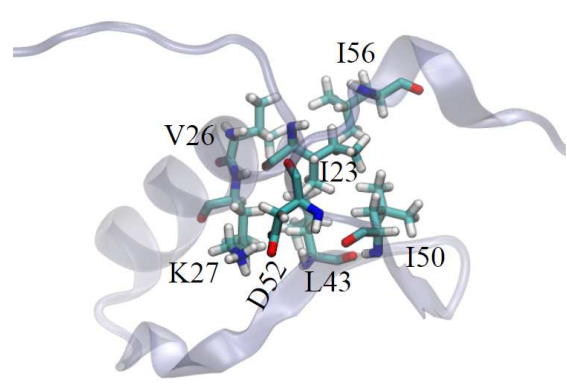

b

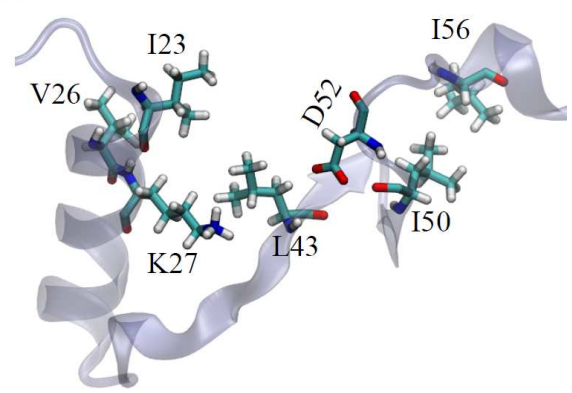

Fig. S2 The AFM-like unfolding of the Ubiquitin core. (a) The initial state with various native contacts; (b) The final state where all native contacts were lost.

Figure S2 highlights the unfolding process related to the third force peak (orange line; at $110 \AA$ ) in Fig. 3a. To break native contacts in the Ubiquitin core, the force of about $300 \mathrm{pN}$ is required to break the salt-bridge (between $\mathrm{K} 27$ and D52) and hydrophobic interactions (involving $123, \mathrm{~V} 26, \mathrm{~L} 43,150$ and 156 ).

\section{Comparison between two simulations with different pulling rates}

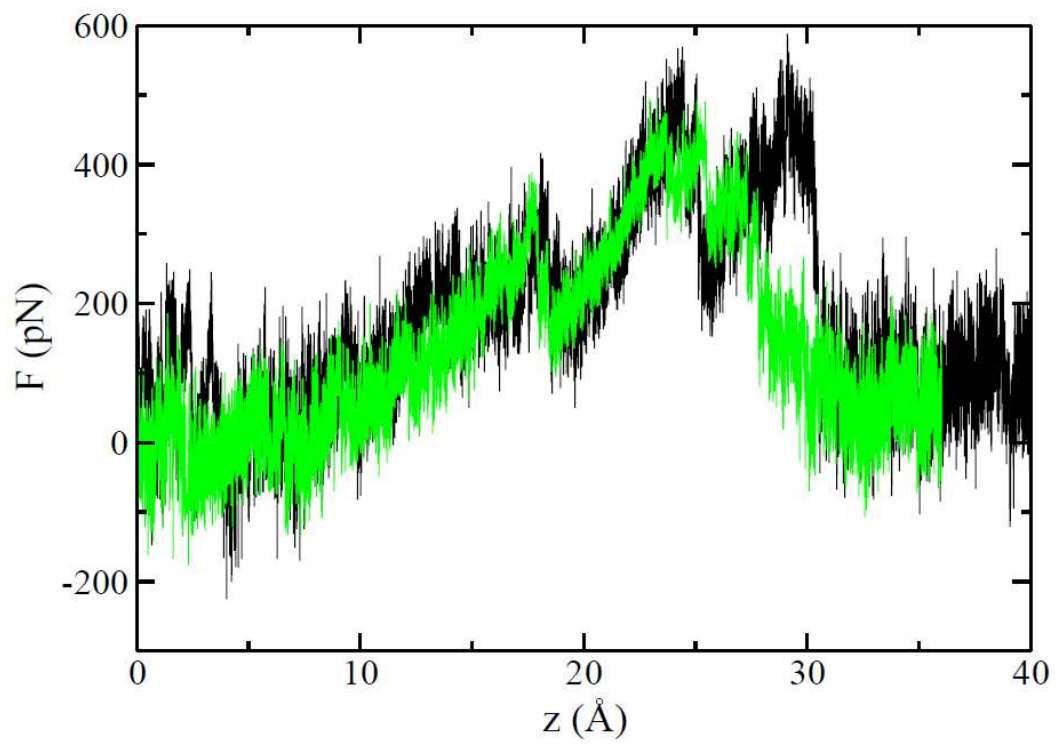

Fig. S3 Position-dependent pulling forces for two different pulling velocities. $v=0.1 \AA / n s$ (green) and $1.0 \AA$ 战 (black). 
Figure S3 shows pulling forces at different pulling stages for two different pulling velocities, when pulling out the $\beta$-strand in the C-terminal. In Fig. S3, positions of "valleys" indicate where metastable states are, while positions of "peaks" (followed by sudden drops of forces) indicate where these meta-stable states are about to be broken. We found that positions of "valleys" from these two pulling simulations match each other, suggesting the similar metastable states or unfolding pathways. This was further confirmed from analyses of two simulation trajectories.

\section{Movie files showing simulation trajectories}

AFM.mpg: showing the unfolding process of the Ubiquitin in the AFM-like pulling.

Protease.mpg: showing the unfolding process of the Ubiquitin in the protease-like pulling. 